Conclusion Decades after children and youths are placed in out-of-home care; they are still likely to report worse health than children who grew up in a parental household. Future work from this project will investigate how much of these associations are due to socio-demographic selection into different care experiences, compared to effects of being in care.

\section{P22 ASSOCIATIONS BETWEEN SOCIAL CAPITAL AND MENTAL HEALTH IN TWO RUSSIAN CITIES}

${ }^{1} \mathrm{~N}$ Bobrova*, ${ }^{1} \mathrm{~S}$ Cook, ${ }^{2} \mathrm{AV}$ Kudryavtsev, ${ }^{3,4} \mathrm{~S}$ Malyutina, ${ }^{3} \mathrm{M}$ Voevoda, ${ }^{1,5} \mathrm{DA}$ Leon. ${ }^{1}$ Department of Non-communicable Disease Epidemiology, London School of Hygiene and Tropical Medicine, London, UK; ${ }^{2}$ Central Scientific Research Laboratory, Northern State Medical University, Arkhangelsk, Russia; ${ }^{3}$ Research Institute of Internal and Preventive Medicine - Branch of ICandG SB RAS, Novosibirsk, Russia; ${ }^{4}$ Novosibirsk State Medical University, Ministry of Health of Russia, Novosibirsk, Russia; ${ }^{5}$ Department of Community Medicine, Arctic University of Norway, UiT, Tromso, Norway

\subsection{6/jech-2018-SSMabstracts. 148}

Background Social capital has been extensively studied as one of the determinants of population health including mental health over the past 20 years. There is existing evidence linking depressive disorders with CVD mortality and all-cause mortality in many countries including Russia. However, research exploring determinants of mental health in Russia including social capital is limited.

Methods We assessed relationships between social capital and mental health in a large cross-sectional study, the International Project on Cardiovascular Disease in Russia (IPCDR), based in two Russian cities: Arkhangelsk and Novosibirsk. The study used a random sample of 5051 people aged 35-69. The collected data included measures of socio-economic status and demographic variables: age, sex, education, perceived financial situation, and marital status.

Mental health measures Having any depressive or anxiety disorder were defined as $\geq 10$ score calculated from the Patient Health Questionnaire (PHQ-9) and Generalized Anxiety Disorder 7 (GAD-7) scales.

Social capital measures We used three questions to measure social capital with 11-point scale answer options: 1) Social mistrust, 'Generally speaking, would you say that most people can be trusted, or that you can't be too careful in dealing with people?'; 2) Perceived lack of fairness: 'Do you think that most people would try to take advantage of you if they got the chance, or would they try to be fair?'; and 3) Perceived unhelpfulness: 'Would you say that most of the time people try to be helpful or that they are mostly looking out for themselves'. The scales were dichotomised 0/6-low social capital and 7/10-high social capital and each was used as a separate dependent variable in analysis.

Statistical analysis We employed two logistic regression models to assess associations between social capital and mental health, 1) adjusting for age, sex and city, 2) adjusting for age, sex, city, marital status, education level and self-reported financial situation.

Results All three measures of social capital were associated with depression and anxiety after adjusting for socio-economic and demographic variables: 'Social mistrust': OR 1.49 (95\% CI, 1.14 to 1.94), 'Perceived lack of fairness': OR 1.75 (95\% CI, 1.39 to 2.20), 'Perceived unhelpfulness' OR 1.72 (95\% CI, 1.36 to 2.17) for depressive disorder; 'Social mistrust': OR 1.40 (95\% CI, 0.99 to 1.99), 'Perceived lack of fairness':
OR 2.37 (95\% CI, 1.71 to 3.29), and 'Perceived unhelpfulness': OR 2.19 (95\% CI, 1.57 to 3.04) for anxiety disorder. Conclusion Our findings from Russia support previous research showing the relationships between social capital and mental health. Further investigations on direction of the effect and pathways explaining these associations are needed.

\section{P23 'POSITIVE AND NEGATIVE SOCIAL SUPPORT AND HPA- AXIS HYPERACTIVITY: EVIDENCE FROM GLUCOCORTICOIDS IN HUMAN HAIR'}

${ }^{1} \mathrm{E}$ lob*, ${ }^{2} \mathrm{C}$ Kirschbaum, ${ }^{3} \mathrm{~A}$ Steptoe. ${ }^{1}$ Department of Epidemiology and Public Health, University College London, London, UK; ${ }^{2}$ Department of Psychology, Technische Universität Dresden, Dresden, Germany; ${ }^{3}$ Department of Behavioural Science and Health, University College London, London, UK

\subsection{6/jech-2018-SSMabstracts. 149}

Background While positive social support is associated with lower prevalence of disease and better treatment outcomes, negative social relationships can instead have unfavourable consequences for several physical and mental health conditions. However, the specific mechanisms by which this nexus might operate remain poorly understood. Hypothalamic-pituitary-adrenal (HPA) axis hyperactivity owing to psychosocial stress has been proposed as a potential pathway underlying the link between social support and health. Hair glucocorticoids such as cortisol and cortisone are emerging as promising biomarkers of long-term retrospective HPA activation. Therefore, the aim of this investigation was to examine the effects of positive and negative experiences of social support within key relationships (i.e. spouse/partner, children, other family members, and friends) on cortisol and cortisone.

Methods These associations were tested in a sample of 2520 older adults (mean age 68.1) from the English Longitudinal Study of Ageing (ELSA). Hair samples were collected in wave $6(2012 / 13)$. To understand the impact of cumulative exposure to poor social support, the analysis used self-reported data from waves $4(2008 / 09)$ and 6. Both aggregated and sourcespecific effects of social support were tested. Covariates included demographic characteristics, socioeconomic position, health behaviours, and hair characteristics. The analytical strategy was based on multiple linear regression, and missing data were estimated by multiple imputation.

Results In cross sectional analyses, participants who reported lower positive or greater negative social support across all sources had higher levels of cortisol, as well as an elevated cortisol-to-cortisone ratio. Amongst the different components of social support, children exhibited the largest effects on both hormones. In longitudinal analyses, cortisol and the ratio were positively associated with the aggregated scores for cumulative exposure to poor social support, evaluated in retrospect over four years.

Discussion Experiences of low positive and high negative social support, particularly from children, were both related to higher hair glucocorticoid levels. Hence, poorer quality of social relationships in later life may lead to higher psychosocial stress. This in turn could result in HPA-axis hyperactivity and increase the individual's susceptibility to poor health. Health and social care interventions should therefore highlight the importance of improving the quality of social relationships, rather than merely enhancing social interactions, in order to reduce stress and ameliorate health. 